\title{
Emerging role of clinical mass spectrometry in pathology
}

\author{
Angela W.S. Fung, ${ }^{1,2}$ Vijithan Sugumar, ${ }^{3}$ Annie He Ren, ${ }^{3}$ Vathany Kulasingam (i) 3,4
}

'Department of Pathology and Laboratory Medicine, St Paul's Hospital, Vancouver, British Columbia, Canada

2Department of Pathology and Laboratory Medicine, The University of British Columbia, Vancouver, British Columbia, Canada

${ }^{3}$ Department of Laboratory Medicine and Pathobiology, University of Toronto, Toronto, Ontario, Canada

${ }^{4}$ Clinical Biochemistry, University Health Network, Toronto, Ontario, Canada

\section{Correspondence to}

Dr Vathany Kulasingam, Clinical Biochemistry, University Health Network, Toronto, ON M5G 2C4, Canada; Vathany. kulasingam@uhn.ca

Received 14 October 2019 Accepted 14 October 2019 Published Online First 5 November 2019

Check for updates

(c) Author(s) (or their employer(s)) 2020. № commercial re-use. See rights and permissions. Published by BMJ.

To cite: Fung AW.S, Sugumar $\mathrm{V}$, Ren $\mathrm{AH}$, et al. J Clin Pathol 2020;73:61-69.

\section{ABSTRACT}

Mass spectrometry-based assays have been increasingly implemented in various disciplines in clinical diagnostic laboratories for their combined advantages in multiplexing capacity and high analytical specificity and sensitivity. It is now routinely used in areas including reference methods development, therapeutic drug monitoring, toxicology, endocrinology, paediatrics, immunology and microbiology to identify and quantify biomolecules in a variety of biological specimens. As new ionisation methods, instrumentation and techniques are continuously being improved and developed, novel mass spectrometry-based clinical applications will emerge for areas such as proteomics, metabolomics, haematology and anatomical pathology. This review will summarise the general principles of mass spectrometry and specifically highlight current and future clinical applications in anatomical pathology.

\section{INTRODUCTION}

\section{Introduction to clinical mass spectrometry}

Mass spectrometry (MS) is a powerful analytical technique that measures the mass to charge ratio $(m / z)$ of one or more molecules in a sample, where $m$ is the molecular mass in Daltons and $z$ is the amount of charge present on the ion. MS can be used for accurate identification of unknown analytes, quantification of known compounds, and determination of structural and chemical properties of molecules. Compared with established methods such as immunoassays, MS can detect multiple analytes of interest in a high-throughput fashion at improved speed and specificity. ${ }^{1}$ Over the past two decades, the technology has become considerably more robust, accurate and cost-effective, leading to the steady adoption of MS in all subdisciplines of laboratory medicine.

With increasing clinical applications of MS, there are a number of reviews published on this topic, particularly focusing on clinical chemistry and more recently microbiology and haematology. ${ }^{1-4}$ Although molecular imaging of human tissue using MS has been described for well over a decade, its application in anatomical pathology is only now at the cusp of emergence. The main objectives of this review are to outline the general principles of MS and its instrumentation, as well as to summarise the current and evolving clinical applications of MS with a spotlight on anatomical pathology.

\section{Principles of MS}

The basic principles of MS analysis are based on the production, selection, fragmentation and detection of charged ion species from analytes of interest in a sample. MS identifies and quantifies molecules by measuring their $m / z$. The process of MS analysis involves an initial ionisation step in an ion source, where positive or negative charged ions are produced from neutral molecules. The ions of interest are then selected in a mass analyser according to their $\mathrm{m} / \mathrm{z}$ ratios, and subsequently detected on a mass detector. The specific ion of interest is plotted in a mass spectrum, where the relative abundance or intensity of the ion is plotted against $m / z$.

As the analytes travel through various regions of the mass spectrometer, fragmentation may occur. Since fragmentation occurs at specific bonds depending on the chemical nature and strength of the bond, each molecule produces a characteristic mass spectral fingerprint that is related to its molecular structure. Typically, low levels of ion source fragmentation are preferable for molecular mass determination, whereas higher levels of ion source fragmentation are preferable for molecular structure analysis. In tandem MS (MS/MS), two or more mass spectrometers are coupled together with various fragmentation techniques to additionally separate and identify molecular ions that have similar $m / z$ ratios. The specific pair of $m / z$ values of the precursor and product ions is termed a 'transition'. The peak area of the extracted ion chromatogram of an analyte of interest, identified by its $m / z$ transition, can be integrated and calculated for quantitation using an internal standard. An internal standard is usually an isotopically labelled version of the molecule of interest that is added in constant known amounts to the samples at the beginning of the workflow. By analysing the ratio of the peak area associated with the analyte of interest and the internal standard, the exact concentration of the analyte can be calculated. When a single transition is monitored, it is termed 'selected reaction monitoring (SRM)', and when multiple transitions are monitored it is termed 'multiple reaction monitoring (MRM)'.

\section{Instrumentation for MS}

When selecting an MS instrument, mass accuracy and resolution are desirable properties in MS analysis. High resolution increases mass accuracy and reduces interference with adjacent peaks from molecules of similar mass, thereby achieving a higher specificity. There are numerous variations in mass spectrometer instrument configurations. A typical mass spectrometer can be divided into three main components: (1) ion source, (2) mass analyser and (3) mass detector. In this section, different types of each component and common MS instruments used in the clinical laboratory will be briefly discussed. 
The ion source is where positive or negative ions are produced from a neutral analyte of interest, a process known as 'ionization'. The choice of ion source used in the clinical laboratory depends on the physical properties of the molecules being analysed and the desired output of ionisation. In the clinical laboratory, electrospray ionisation (ESI) and atmospheric pressure chemical ionisation (APCI) are the two most common soft ionisation techniques used for quantitative analysis by liquid chromatography tandem mass spectrometry (LC-MS/MS) at atmospheric pressure. ESI is an efficient ionisation technique and does not produce excessively fragmented molecules, which makes it particularly useful for small molecules, proteins and peptides. APCI is considered a harsher ionisation technique compared with ESI as it may cause loss of modifications of target compounds (eg, glucuronidation); however, it is more efficient in ionising small, heat-stable molecules and removing matrix components for analytes such as steroids and some drug molecules. Another commonly used ionisation method in the clinical laboratory is the matrix-assisted laser desorption/ ionisation (MALDI). MALDI is a type of soft ionisation that produces single charged species and can analyse a variety of molecules including nucleic acid, peptides and proteins.

Once ions are generated in the ion source, the mass analysers separate and fragment ions of specific $m / z$ to be detected. Quadrupole mass spectrometers are the most widely used mass analysers in clinical laboratories, where four parallel electrically conductive rods form a channel for ions to pass through from one end to another. To select a specific $m / z$ ion to pass through to the detector, a combination of direct current potentials with oscillating radiofrequency is applied to the quadrupole rods causing the ions within a narrow $m / z$ range to stay in the channel and the ions outside the range to be ejected radially. This is analogous to monochromator filters in optical techniques, and often referred as the 'mass filters'. Many clinical qualitative and quantitative methods use quadrupole mass spectrometers, including drugs, ${ }^{5-10}$ steroids, ${ }^{11-19}$ small molecules and metabolites, ${ }^{20-24}$ as well as peptides and proteins. ${ }^{25-29}$ Another commonly used mass analyser is the time-of-flight (ToF) mass analysers. In ToF mass analysers, ions travel down the flight tube accelerated by applied potential. The flight time, or the time it takes for the ion to reach the detector, is related to the $m / z$ ratio of the ion, where lighter ions travel faster and reach the detector before a heavier ion does. ToF-MS has several advantages, including higher dynamic range, mass accuracy, and acquisition speed with moderate resolution and sensitivity. Notable clinical applications of ToF-MS include identification of microbials (via MALDI-ToF) ${ }^{30} 31$ and genotyping. ${ }^{32-34}$

When multiple mass analysis occurs in series, it is known as tandem mass spectrometry (MS/MS). Various configurations that include arranging identical mass analysers in tandem (eg, triple quadrupole or $\mathrm{ToF} / \mathrm{ToF}$ ) or hybrid combinations of different mass analysers (eg, quadrupole-orbitrap) have been designed for several applications. MS/MS methods are useful for structural analyses and quantitation of compounds that have similar structure and relative mass. LC-MS/MS is a dominant MS-based technique used in clinical laboratories for quantitative analysis due to its high selectivity, ability to detect low concentrations of analytes and ability to measure multiple analytes in a single method (multiplex).

\section{CURRENT CLINICAL APPLICATIONS OF MS IN CLINICAL LABORATORIES}

One of the first uses of MS in clinical laboratories is in the area of toxicology. Confirmatory urine drug screens for clinical or forensic purposes are commonly measured by gas chromatography-MS (GC-MS), and more recently LC-MS/
MS. The predictable and consistent hard ionisation fragmentation patterns produced by electron ionisation followed with GC-MS allow for the identification of unknown compounds by matching the full mass spectrum of the sample with a library or database. An important limitation of GC-MS is the requirement of compounds to be sufficiently volatile. This requires samples with poor volatility to be derivatised during sample preparation, where the analytes are chemically altered to be more suitable for analysis.

LC-MS/MS is the most commonly used method for many clinical applications today, ranging from qualitative urine drug screens to quantitative, high-throughput and esoteric analyses including therapeutic drug monitoring (eg, immunosuppressant drugs, antiretroviral drugs, antiepileptic), ${ }^{5-10}$ steroids (vitamins, testosterone, androstenedione, 17-hydroxyprogesterone, aldosterone, cortisol, oestrogen), ${ }^{11-18}$ inborn errors of metabolism and newborn screening (eg, amino acids, carnitine and acylcarnitine), ${ }^{23} \quad 35-39$ endocrinology (eg, biogenic amines, 5-hydroxyindolacetic acid, insulin, thyroglobulin) 25264041 and immunology (eg, monoclonal immunoglobulins, serum free light chains, IgG subclasses ${ }^{27-29} 42$ ). Please see recent reviews for more details. ${ }^{133}$ MALDI-ToF MS has also recently been in development for qualitative and quantitative peptide and protein assays. Examples include monoclonal immunoglobulins and serum free light chains, ${ }^{29} 4244$ haemoglobin A1c, ${ }^{45}$ insulin-like growth factor- $1,{ }^{46}$ multiplex biomarker panel of $\mathrm{C}$ reactive protein, serum amyloid $\mathrm{A}$, faecal calprotectin and cystatin $\mathrm{C}$. $^{47}$

In microbiology, quantitative measurements of antibiotics and antifungal drugs are available. ${ }^{1048} 49$ Recent implementation of MALDI-ToF MS has revolutionised the workflow for the identification of micro-organisms. The identification is done by matching the ionised proteins and peptides that are specific to the organism and present in cultures to a validated spectral library. Compared with conventional microbiological techniques (eg, Gram stain, culture, biochemical tests and susceptibility testing), MALDI-ToF MS offers significantly shorter turnaround time in terms of species identification. A limitation of MALDI-ToF MS, however, is its relative lack of specificity in differentiating closely related strains of bacterial species. In molecular diagnostics, genotyping and mutational analysis in liquid biopsy have been in development using MALDI-ToF MS. ${ }^{32-34} 50$ Please see recent review on other clinical and near clinical applications of MALDI-ToF MS for details. ${ }^{51-53}$

\section{EMERGENCE OF MS APPLICATIONS FOR ANATOMICAL PATHOLOGY \\ Omics}

Novel applications of MS methods are driving omics research (lipidomic, metabolomic and proteomic) and biomarker discovery in a variety of specimen types including blood, urine and biopsy tissues. Proteomic analysis using MS can be approached using two methods: top-down proteomics and bottom-up proteomics. Top-down proteomics involves the intact analysis of the entire protein. However, many proteins involved in human diseases share similar masses, making the top-down method ineffective for distinct clinical subtyping. The top-down proteomics method is also unable to accurately determine the prevalence of different protein variants if they possess similar masses. In contrast, bottom-up proteomics involves enzymatic cleavage of the whole protein through peptidase digestion to generate a mixture of peptides. A commonly used peptidase is trypsin, where it cleaves proteins at predictable sites (the carboxyl side of the amino acids lysine and arginine, except when they are followed by proline), 
and the peptide mixture generated from trypsin digestion is a characteristic signature of the precursor protein. The peptide mixture can then be purified through chromatography and other preparatory methods prior to MS analysis. The separation of the digested protein through chromatography and use of MS to analyse the identity and prevalence of proteins constitute a more effective method of protein identification for clinical diagnosis.

In anatomical pathology, whole proteome profiling from tissue slides is beginning to be established to examine global protein expression patterns and elucidate the pathophysiology of diseases. For instance, routine pathological diagnosis of renal diseases requires interpretation of morphological alterations observed by light microscopy (LM), immunofluorescence (IF) and electron microscopy (EM), with correlation to clinical parameters. Immunohistochemical (IHC) staining of proteins has additionally been used to identify the protein of interest. In difficult or equivocal cases, more sensitive techniques such as immunoelectron microscopy (IEM) and laser microdissection mass spectrometry (LMDMS) analyses may assist in the confirmation of identity and localisation of renal protein deposits. ${ }^{5-56}$ In particular, progress has been demonstrated in the clinical utility of amyloid and kidney proteome analysis, which holds importance for diagnosing amyloidosis and characterisation of renal diseases. 555758

\section{Amyloidosis}

Amyloidosis is a group of diseases that result from build-up of insoluble protein aggregates in various tissue and organs. These proteins, known as amyloid fibrils, are abnormal in structure due to protein misfolding and accumulate extracellularly in $\beta$-pleated structures. Amyloid deposits are characterised by their ability to develop both systemically and locally in specific sites of the body, rendering increased cellular toxicity and organ damage. ${ }^{59}$ The diagnosis of amyloidosis presents a unique challenge as the clinical presentation is often subtle and may overlap with several different conditions. Clinical management depends on the disease phenotype, which depends on the protein composition of the amyloid fibrils. For example, hereditary transthyretin amyloidosis and immunoglobulin-associated amyloidosis, both present with similar clinical syndrome, are managed differently with liver transplantation or stem cell transplantation/chemotherapy, respectively. ${ }^{60}$ Accurate and comprehensive subtyping of amyloid diseases is critical for appropriate clinical management, since there are distinct treatment options for the different subtypes. To date, there are over 36 different subtypes of localised and systemic amyloidosis syndromes. ${ }^{61} 62$

For diagnosis, the presence of amyloid deposits in tissue biopsy is first confirmed. On light microscopy, amyloid deposits can be found in all compartments, are acellular and have a characteristic positive Congo red stain, where amyloid deposits show reddishbrown and apple-green birefringence under polarised light. On EM, amyloid fibril deposits are solid, non-branching and randomly arranged, with a diameter ranging from 7 to $14 \mathrm{~nm} .{ }^{63}$ Then, the protein composition (subtype) of the amyloid deposits is determined via a variety of techniques. For immunoglobulin-associated amyloidosis, intense staining for immunoglobulin light chains and/ or heavy chains on IF (for renal and IHC for all other organs) is diagnostic for immunoglobulin light chain (AL), immunoglobulin heavy chain $(\mathrm{AH})$ and immunoglobulin heavy and light chain (AHL) amyloidosis. ${ }^{63}$ IHC has also been used for diagnosis of serum amyloid A (AA) amyloidosis and LECT2 amyloidosis. ${ }^{55}$ These conventional amyloidosis subtyping techniques based on antibodies often have poor specificity and sensitivity due to high background staining caused by serum contamination, ${ }^{60}$ loss of epitope recognition from formalin fixation-induced protein crosslinking, and dependency on using multiple and specific antibodies to recognise all potential epitopes for all various amyloid subtypes. ${ }^{59}$ The inherent limitations of antibody-based techniques led to the development of novel subtyping methods such as LMD-MS. In LMD-MS, a laser is coupled to a light microscope and used to selectively dissect a subpopulation of protein aggregates of interest from the tissue on the slide. The protein mixture is denatured, digested with trypsin and analysed via LC-MS/MS. The tandem mass spectra are then searched against different search engines for protein identification and semiquantitative measure of its relative abundance.$^{64}$ LMD-MS has a major advantage over conventional amyloid subtyping where it is a single test that can simultaneously identify and semiquantify the amyloid protein composition, thereby improving the cost-effectiveness and efficiency in amyloid diagnosis. ${ }^{60}$ The novel MS-based method has facilitated accurate identification of rare amyloid types where antibodies may be lacking, such as AGel, AApoAI, AApoAIV AH, ALect2, Alns, ALys and their genetic variants. ${ }^{62}{ }^{65}{ }^{66}$ LMD-MS can identify the subtype of amyloidosis in more than $92 \%-95 \%$ of cases. ${ }^{59} 67-69$ Overall, LMD-MS has higher sensitivity and specificity compared with IF and IHC, and is currently the gold standard for amyloid subtyping. ${ }^{70}$ LMD-MS is indicated for the confirmation of amyloid subtype, when there are equivocal Congo red or heavy and/or light chains staining, and when there are inadequate tissue sample for IF studies, in difficult cases such as differentiating congophilic fibrillar glomerulonephritis (FGN) and amyloidosis, and in rare cases such as familial and hereditary amyloidosis. 55766677071

\section{Fibrillar glomerulonephritis}

FGN is a rare glomerular disease that is associated with autoimmune diseases, viral infections and malignant neoplasm. ${ }^{72}$ The pathogenesis and therapy for FGN remain largely unknown. ${ }^{72}$ The fibrillar protein deposits of FGN are morphologically similar to amyloid fibrils, where they are both solid, randomly arranged fibrils, with FGN having thicker fibrils with diameter ranging from 10 to $30 \mathrm{~nm}^{6372}$ There is an overlap in the thickness of the fibrils, and fibril diameter should not be solely relied on as a distinguishable feature. FGN deposits are found in the mesangium along the capillary walls of the glomeruli. FGN deposits are primarily composed of polyclonal IgG, $\mathrm{C} 3$, and $\kappa$ and $\lambda$ light chains, which can be stained with antisera to immunoglobulins by IF. However, monoclonal FGN had been reported occasionally. ${ }^{73}$ In histology, amyloid deposits are generally identified by their characteristic apple-green colour under polarised light with Congo red staining; meanwhile, FGN deposits are Congo red-negative. Congo red positivity under polarised light is thus often the differentiating factor between amyloid and non-amyloid diseases. However, certain cases of FGN have been shown to be congophilic despite histochemically and proteomically identical to traditional FGN. ${ }^{74}$ For these cases where Congo red is unable to differentiate the diseases, EM becomes a necessity for diagnosis; however, it is often routinely inaccessible. LMD-MS has been used to extract FGN glomeruli and examine the protein composition to search for a suitable diagnostic biomarker. ${ }^{75}$ DnaJ homologue subfamily B member 9 (DNAJB9) was identified as the first histological kidney tissue marker for FGN. ${ }^{7-77}$ Studies showed that DNAJB9 colocalises with IgG deposition by dual IF and localisation of DNAJB9 to individual FGN fibrils by IEM. DNAJB9 IHC studies on kidney biopsy demonstrate high sensitivity (98\%) and specificity (>99\%) for FGN. ${ }^{7677}$ As a result, the DNAJB9 IHC test is currently available in some clinical laboratories to reduce reliance on Congo red staining and inaccessible EM, which significantly simplify the diagnosis of 
FGN. ${ }^{76}$ Through these LMD-MS-based studies, a new variant of FGN termed 'heavy chain fibrillary glomerulonephritis' was also identified, which is characterised by Congo red-negative, DNAJB9negative fibrillar deposits consist of heavy chain immunoglobulins that appear to be distinct from typical FGN both pathogenetically and clinically. ${ }^{72} 7678$ More recently, serum levels of DNAJB9 were measured by immunoprecipitation-based MRM and demonstrated to be elevated in patients with FGN when compared with nonFGN glomerular diseases, such as immunoglobulin light chain amyloidosis or multiple myeloma, with adjustment for estimated glomerular filtration rate (eGFR) differences. ${ }^{79}$ Serum DNAJB9 predicted FGN with a moderate sensitivity (67\%) and high specificity $(98 \%)$ in a discovery cohort. ${ }^{79}$ Further studies are needed to elucidate new variants of FGN, clinical utility of serum DNAJB9 as a non-invasive FGN biomarker, pathogenetic role of DNAJB9 and possibility as a targeted therapy. ${ }^{72}$ Overall, LMD-MS is indicated to help differentiate difficult cases, such as when there are equivocal Congo red staining, in the presence of heavy chain component to distinguish monoclonal heavy chain FGN from $\mathrm{AH}$ amyloidosis and heavy chain deposition disease (HCDD), and differentiating congophilic FGN from amyloidosis, FGN with concurrent renal diseases (IgA, membranous and diabetic nephropathy), and FGN from immunotactoid glomerulopathy (ITG) associated with chronic lymphocytic leukaemia (CLL). 55278

\section{Immunotactoid glomerulopathy}

ITG is another rare glomerular disease that is frequently associated with monoclonal gammopathies and hypocomplementaemia, but not cryoglobulinaemia and autoimmune diseases. ${ }^{80}$ The pathogenesis of ITG is largely unknown, and treatment depends on the underlying haematological process. ${ }^{80}$ In pathology, ITG is characterised by proliferative glomerulonephritis pattern by LM, monotypic IgG (>90\% cases) with $\kappa$ or $\lambda$ light chain restriction and C3 staining by IF, and protein deposits defined by microtubular structure with distinct hollow centres measuring $10-90 \mathrm{~nm}$ in diameter deposited in organised, parallel arrays in the mesangium, subendothelial and subepithelial space of the glomeruli by EM. ${ }^{6381}$ LMD-MS confirmed the presence of monotypic immunoglobulins, and the activation of classical and terminal pathways of complement as C3 and C4 was identified in ITG cases.$^{80}$ Comparing the kidney proteome profiles of amyloidosis, FGN, ITG and cryoglobulinaemic glomerulonephritis by LMD-MS in a discovery cohort, apolipoprotein E (apo E) was hypothesised to be required for fibrillogenesis, where the size and organisation of the protein deposits (ie, fibrillar vs microtubular) are associated with the ratio of apo E to immunoglobulin/amyloidogenic protein. ${ }^{57}$ Overall, LMD-MS for ITG is indicated to confirm the identity of protein deposits and to distinguish difficult cases. For instance, ITG associated with CLL typically has small microtubules that may not show the distinct hollow centres and may resemble FGN fibrils even by EM, and LMD-MS proteomic analysis can support the differential diagnosis. $^{82}$

\section{Monoclonal immunoglobulin deposition disease and proliferative glomerulonephritis with monoclonal IgG deposits}

Non-organised monoclonal immunoglobulin deposits are seen in monoclonal immunoglobulin deposition disease (MIDD) and proliferative glomerulonephritis with monoclonal IgG deposits (PGNMID). MIDD is classified into three subtypes depending on the composition of deposits: light chain deposition disease, HCDD, and light and heavy chain deposition disease. The light chain deposition disease is the most common subtype. ${ }^{58}$ On LM, MIDD most commonly shows nodular sclerosing lesions, although rare cases have membranoproliferative and mesangial proliferative pattern of injury, and a thickened glomerular and tubular basement membrane. ${ }^{58}$ Sometimes MIDD nodules may appear similar to diabetic mesangial nodules when IF staining for immunoglobulins is ambiguous. ${ }^{55}$ On IF, diffuse linear staining of the heavy or light chain immunoglobulins along the glomeruli, tubules and vascular myocytes is observed, depending on the subtype. ${ }^{63}$ On EM, MIDD shows a characteristic punctate granular deposits in the mesangium, and along the glomerular and tubular basement membrane. ${ }^{70}$

Meanwhile, the majority of PGNMID shows a membranoproliferative or endocapillary proliferative glomerulonephritis pattern on LM. On IF, PGNMID shows restricted staining for a single light chain isotype ( $\kappa$ more common than $\lambda$ ) and a single heavy chain subtype. Monoclonal IgG3 is the most common deposit in PGNMID, although rare cases have been reported which include other IgG subtypes, IgA, IgM or light chains. ${ }^{83-85}$ On EM, PGNMID shows non-organised granular deposits in the subendothelial and mesangial space. ${ }^{63}$

MIDD and PGNMID differ in their deposit localisation and composition. The deposits in MIDD can been found in glomerular, tubular and vascular regions, whereas PGNMID deposits are confined to the glomeruli. ${ }^{70}$ PGNMID deposits are typically intact immunoglobulins, whereas the heavy chains in MIDD are typically composed of truncated IgG1 that lacks the $\mathrm{CH} 1$ domain. ${ }^{70}$ Both can rarely also be IgA or IgM or light chain driven. ${ }^{63} 70$ Patients with PGNMID with monoclonal IgG3 deposits are less likely (30\%) to have detectable serum monoclonal proteins compared with IgG1 and IgG2 subclasses. ${ }^{5486}$

Diagnosis for both MIDD and PGNMID depends heavily on IF staining of immunoglobulins. However, it is important to note that occasionally the degree of immunoglobulin deposition in the glomerulus may be below the detection threshold, epitopes may be masked, or antibodies unavailable due to extremely rare heavy chains such as $\operatorname{IgD} .^{547-90}$ There are also reports of discrepancies between immunoglobulins identified in serum and/or urine studies (ie, serum and urine protein electrophoresis, immunofixation, and serum free light chain studies) and immunoglobulins identified on renal biopsy by IF, which presents diagnostic challenges. ${ }^{5491}$ Therefore, LMD-MS is particularly useful to confirm the presence of immunoglobulin heavy and light chains when IF studies are equivocal or unavailable (eg, IgG subclasses, IgD HCDD), and when immunoglobulins in serum and/or urine identified were discordant with renal deposits identified by IF..$^{548790}$

\section{C3 glomerulopathy: C3 glomerulonephritis and dense deposit disease}

C3 glomerulopathy is a rare disease that is caused by dysregulation of the alternative complement pathway and is characterised by renal deposits of complement factors from the alternative and terminal pathways with minimal or no immunoglobulins. ${ }^{556392} \mathrm{C} 3$ glomerulopathy comprised two pathological entities: $\mathrm{C} 3$ glomerulonephritis (C3GN) and dense deposit disease (DDD). On LM, both C3GN and DDD are characterised by mesangial proliferative, membranoproliferative or endocapillary proliferative glomerulonephritis. ${ }^{63}$ On IF, bright C3 staining with at least two orders of magnitude in dominance and an absence of immunoglobulin staining is expected. ${ }^{92}$ However, there are reports of masked monotypic immunoglobulin deposits in paraffin-embedded tissues, where negative staining of immunoglobulins was observed on IF with positive staining of immunoglobulins revealed on protease digestion. ${ }^{88} 89$ Additionally, approximately $30 \%$ of postinfectious glomerulonephritis are $\mathrm{C} 3$-dominant on renal biopsy, although it is characterised by codeposition of $\operatorname{IgG}$ and $\mathrm{C} 3 .^{92} \mathrm{C} 3 \mathrm{GN}$ and DDD 
are best distinguished by their ultrastructure on EM. C3GN is characterised by moderate electron dense, ill-defined granular deposits in the mesangium, subendothelial and/or subepithelial space, and DDD is characterised by highly electron dense, osmiophilic 'sausage-like' intramembranous deposits and mesangial rounded nodular deposits. ${ }^{6370}$ Subepithelial large 'humps' may occur in either lesions. ${ }^{70} 92$ LMD-MS studies have enabled detailed analysis on the previously unknown protein composition of the deposits in C3 glomerulopathies. ${ }^{93}{ }^{94}$ Surprisingly, both C3GN and DDD have similar protein compositions from the alternative and terminal complement pathway, where $\mathrm{C} 3$ and cleavage product C3dg were present as the predominant glomerular complement proteins and lesser amounts of C9, C5, C6, C7 and C8 were also detectable..$^{9394}$ Factor H-related proteins (FHR1-5) and fluid-phase regulators of the terminal complement complex (clusterin and vitronectin) were also identified..$^{93}$ There was a lack of complement proteins from the classical pathway, such as $\mathrm{C} 1, \mathrm{C} 2$ or $\mathrm{C} 4 .{ }^{93}$

Dysregulation of the alternative complement pathway may be due to primary causes such as de novo mutations in complement activating and regulating proteins or due to secondary causes that result in overactivation of the pathway. ${ }^{58}$ There is a high prevalence $(60 \%-80 \%)$ of patients aged over 50 years old with C3 glomerulopathy and concurrent monoclonal gammopathy at the time of diagnosis, which far exceeds the prevalence of monoclonal gammopathy of unknown significance and C3GN for aged less than 50 years old. ${ }^{89}{ }^{95}{ }^{96}$ Monoclonal immunoglobulins have been demonstrated to act as C3 nephritic factor or factor $\mathrm{H}$ autoantibody, which results in the overactivation of the alternative pathway of complement. ${ }^{97-100}$ The subtype of ' $\mathrm{C} 3$ glomerulopathy with monoclonal gammopathy' is coined to distinguish C3 glomerulopathy deposits with little to no immunoglobulins in the setting of monoclonal gammopathy, where the monoclonal immunoglobulin contributes to the dysregulation of alternative complement pathway. The high prevalence of C3 glomerulopathy with monoclonal gammopathy and the potential of masked or codeposition of immunoglobulin can present diagnostic challenges to C3 glomerulopathies. Thus, LMD-MS studies are indicated when there are equivocal IF staining for immunoglobulins and $\mathrm{C} 3$, and to confirm the cause and protein deposits profile for proliferative glomerulonephritis (immunemediated vs complement-mediated). ${ }^{55}$

\section{MS imaging}

Another emerging technology that has potential clinical applications in pathology is MS imaging (MSI), which employs MS to map the spatial distribution of molecules in a thin tissue sample section. The gold standard of diagnosis for tissue samples has traditionally been a combination of chemical staining and light microscopy, with the results being interpreted by a skilled anatomical pathologist. MSI, however, is remarkable for its ability to image thousands of molecules, such as lipids, proteins, peptides, drugs and metabolites in tissues. MSI also possesses practical advantages compared with current methodology, including analysing tissue directly (in situ), identifying endogenous biomolecules and exogenous compounds in a label-free and multiplex manner, and at the same time correlating molecular spatial distribution to traditional histology. The technique allows for an understanding of the molecular basis and mechanism of disease with relation to tissue morphology. Clinical applications for MSI have been proposed to include tumour typing and disease staging, ${ }^{101}$ tumour margin assessment, intraoperative tumour excision, ${ }^{102}$ drug localisation ${ }^{103} 104$ and biomarker discovery. ${ }^{101} 105$

MSI by MALDI-ToF MS was first described by Caprioli and colleagues $^{106}$ over two decades ago. MSI can be performed on fresh/frozen ${ }^{107-109}$ and formalin-fixed paraffin-embedded (FFPE) tissues. ${ }^{110-113}$ While a wide range of biomolecules, such as peptides, lipids, glycans, small molecules and metabolites, can be detected on FFPE tissues, only lipids and intact proteins are detected on fresh/frozen tissues. ${ }^{114-116}$ Fresh/frozen tissues require immediate freezing, cryosection to micrometre-thick slices, and being subsequently thaw-mounted to a slide. ${ }^{117}$ FFPE tissues require additional sample preparation steps to remove paraffin prior to MSI analysis, and as such may introduce interlaboratory variations as small compounds may be stripped concomitantly. ${ }^{115}$ Nevertheless, analysis of FFPE tissues offers unique advantages, as it allows access to a large repertoire of archived tissues with well-documented clinical information and correlation to IHC and molecular data. Additional sample preparation steps can also be intentionally used to enhance signal and increase specificity for the analyte of interest, such as on-tissue chemical derivatisation or enzymatic treatment for imaging of testosterone, ${ }^{118} 2$-picolylamine, ${ }^{119}$ neurotransmitters, ${ }^{120} \mathrm{~N}$-glycans ${ }^{121}$ and hexosylceramides. ${ }^{122}$

In addition to tissue types, the method and instrumentation for MSI analysis should be considered, including factors such as image resolution, speed of analysis and the availability of clinically validated diagnostic biomarker libraries. Particularly, there is a balance and trade-off in terms of image spatial resolution and analysis speed. The higher the spatial resolution desired for imaging application, the slower the pixel-by-pixel sampling speed for enhanced analyte extraction. The availability of clinically validated diagnostic biomarker libraries can significantly impact interlaboratory variation and allowed for standardised, reproducible interpretation and reporting of pathology results. Therefore, image resolution, speed of analysis and availability of validated database directly impact clinical utility and usefulness of MSI.

One of the most common and well-studied mass spectrometers for MSI is the MALDI-ToF. Under vacuum, an ultraviolet or infrared laser irradiates the matrix cocrystallised tissue sample to desorb and ionise the analytes of interest in a raster-stepped sampling of the entire tissue surface. A mass spectrum is generated per given tissue coordinates or pixel. When regions of interest or the whole tissue is measured, a two-dimensional ion intensity map is generated pixel-by-pixel from the mass spectra obtained from the specific tissue coordinates. If serial tissue sections are analysed, advanced imaging software can generate a threedimensional image of the ion intensity map. One of the limitations of MALDI-ToF MSI is that additional sample preparation is required, where the prepared tissue is coated with a MALDI matrix-an organic acid chemical compound that cocrystallises and extracts the analyte during the pulsed laser ionisation process. The quality of the matrix deposition and its application technique have significant effects on the analytical sensitivity (ie, signal to noise ratio) and spatial resolution of the MS image. The matrix is selected based on the physical and chemical properties of the molecule of interest. Three common matrices used for peptides, lipids and other low molecular weight molecules are $\alpha$-cyano-4-hydroxycinnamic acid (CHCA), sinapinic acid (SA) and 2,5-dihydroxybenzoic acid (DHB). CHCA and SA are often used for peptides, SA and DHB are generally used for proteins, while DHB is useful for carbohydrates and lipids. ${ }^{117} 123$ There are also new matrices developed for specific classes of molecules, such as polar lipids, ${ }^{124}$ non-covalent bound complexes ${ }^{125} 126$ and liquid matrices for improved homogeneity for quantitation. ${ }^{127}$ A homogeneous coating of matrix with small, uniform matrix crystals of low-micrometre dimensions is desirable. Some techniques that employ this feature are high-throughput electrospray, ${ }^{128}$ matrix coating assisted by an electric field ${ }^{129} 130$ and 
sublimation under vacuum to name a few. ${ }^{131} 132$ In consideration for rapid clinical application, matrix precoated glass slides were also developed to increase throughput. ${ }^{133} 134$ Another vacuumbased MSI technique is the secondary ionisation MS (SIMS), which uses a focused ion beam to ionise molecules in the tissue sample. Secondary ions are then generated and analysed by the mass spectrometer. ${ }^{135136}$ MALDI-ToF MSI typically can achieve 10-20 $\mu \mathrm{m}$ spatial resolution; meanwhile, SIMS MSI can achieve $<1 \mu \mathrm{m}$ spatial resolution. ${ }^{137}$ However, the drawbacks of SIMS are that it is a destructive technique that obliterates all molecules that it ionises, has limitations in analysing large intact biomolecules $(>2 \mathrm{kDa})$ and requires complex instrumentation. ${ }^{137}$

\section{Emerging in vivo techniques}

Significant caveats of the current MSI field are the need to process samples for MS analysis, increased turnaround time and limited utility of the technique to assess processed tissue specimens. To enrich the influence of MSI in the clinic, there is a desire for direct, real-time and rapid analysis of unprocessed samples. More recently, atmospheric pressure ambient ionisation MS has gained noteworthy interest for routine clinical use due to significant advantages over other MS techniques, including simpler sample preparation, simpler instrumentation, and analysis in real time in an open environment and at atmospheric pressure, which made the in vivo analysis of tissues using MSI a feasible and attractive option. Since these early experiments in the past decade, more than 40 ambient ionisation techniques have now been described in the literature. ${ }^{138} 139$ Please refer to recent reviews for a summary of common ambient and nonambient ionisation methods for MSI. ${ }^{138} 139$

Rapid evaporative ionisation MS (REIMS) is one of the first ambient ionisation MS techniques that were integrated into routine clinical surgical use. ${ }^{140-142}$ REIMS was originally developed for the accurate identification of cancerous tissues during surgical procedures and has a typical resolution in the range of $0.5-2.0 \mathrm{~mm} .{ }^{143}$ The coupling of the handheld surgical tool and REIMS-MS into a single device led to the creation of the iKnife. ${ }^{141}$ iKnife is a handheld tissue diagnostic device that can immediately analyse, in high resolution and real time, the molecular composition of the tissue it comes into contact with. To do so, REIMS is used to locally heat and disrupt the tissue during electrosurgical procedures, resulting in an aerosol made of tissue-derived gaseous ions being released. The aerosol is subsequently analysed by the mass spectrometer. ${ }^{144}$ Through this rapid REIMS-based analysis, the surgeon is provided with continuous feedback on the composition of the tissue being excised, allowing for the precise removal of any tumour tissue. When comparing in vivo, intraoperative diagnosis made with an iKnife with traditional, postoperative histological diagnosis, the in vivo technique is accurate $92 \%-100 \%$ of the time. ${ }^{141}$ Benefits to using this technique include a lower chance for misidentification of cancer cells during surgical procedures, a reduced risk of resecting healthy tissue and the increased potential to remove virtually all tumour tissues. In addition to differentiating between cancerous and non-cancerous tissues, REIMS-based technologies such as the iKnife can identify different tumour grades, as well as tissues that exhibit precancerous properties within their lipid bilayer. ${ }^{144}$ Moreover, REIMS has been shown to accurately identify histopathological features of a poor prognosis and could potentially be used to identify cancer metastases throughout the body. ${ }^{141} 144$ There is also potential for this technology to be used in preoperative staging of cancer. ${ }^{145}$ The high specificity and wide applications of REIMS have ensured the conception of numerous novel applications for the technology, beyond surgical uses involved with the iKnife.
iEndoscope is another novel technology that stems from REIMS, where the methodology is paired with endoscopic tools for use in diagnostic settings, such as the rapid phenotyping of tissue in rectal and colonic cancers. REIMS equipped with an endoscope allows for visible characterisation of tissue that is typical of the endoscope, while providing additional high-resolution information on tumour biology and chemistry regarding the lesion structure through MS analysis. The primary application of iEndoscope is to accurately differentiate between cancerous and non-cancerous tissues in an endoluminal setting. The endoluminal structure requires the use of an endoscopic polypectomy snare to facilitate the transfer of the aerosol generated by REIMS from the colon to the mass spectrometer. The iEndoscope has been used to identify markers associated with increasing pathogenicity within potentially cancerous tissue. One differentiating factor between REIMS-associated endoscopy and other optical spectroscopic methodologies is its ability to identify abnormal lipid metabolism within the tissue. Abnormal lipid metabolism is often associated with neoplasia, which can facilitate a more confident diagnosis of a cancerous tissue. ${ }^{145}$ Another advantage of REIMS-associated endoscopy is its versatility, where it can be used in adjunct with many different endoscopic imaging tools to provide details related to the biochemistry of the tissue. ${ }^{145}$ Aside from analysing human tissue, REIMS has proved useful for clinical microbiology, where it is able to directly identify bacterial cells within bacterial cell culture without sample preparation. Despite the broad applications of REIMS, be it in the form of the innovative iKnife or iEndoscope, a major disadvantage of REIMS is its destructive nature in both tissue analysis and bacterial cell culture identification, thus limiting the utility of the technique. ${ }^{141}$

The MasSpec Pen is a non-destructive handheld tool designed with three ports centred around the delivery and maintenance of a singular water droplet. The design involves a single water droplet being exposed to the tissue of interest. The droplet acts as the medium used to withdraw analytes, using the premise of liquid-solid chemical extraction. A pressurised vacuum system then moves the water droplet through a port to the mass spectrometer for subsequent analysis. The additional components of the MasSpec Pen include a syringe pump (to eject the water droplet), a small diameter tubing for solvent transport and a probe. The components of the MasSpec Pen must be made of biocompatible materials to ensure both safety and accuracy in in vivo analyses. Hence, polydimethylsiloxane (PDMS), polytetrafluoroethylene (PTFE) and water are the most prominent substances/materials used within the tool. Similar to the iKnife, the MasSpec Pen is highly sensitive and specific for both in vivo and ex vivo analyses and differentiation of tissue types. The MasSpec Pen succeeds in improving on the aforementioned REIMS-based tools in its ability to analyse and identify tissue samples without sample preparation and in real time, while also touting the additional benefit of being a non-destructive method of analysis. ${ }^{146}$

\section{CHALLENGES AND FUTURE APPLICATIONS}

MS has many applications in the clinical laboratory, including clinical chemistry, microbiology, and more recently anatomical pathology, as there are significant advantages for MS-based assays in their multiplexing capacity, high analytical specificity and sensitivity, and potential for real-time in vivo analysis. Prior to clinical implementation of these MS-based methods, a specific gap in clinical need or question needs to be addressed and the advantages and limitations of MS-based methods should be compared with traditional methods in pathology. Additionally, there are desirable and practical features to consider, including high capital costs, requirement of skilled personnel, lack of automation, lack of direct bidirectional interface between MS 
instruments and laboratory or hospital information system, lack of standardisation, and regulatory requirements. Significant progress is being actively pursued by the manufacturers and clinical MS community in regard to regulatory requirements, ${ }^{147} 148$ standardisation of methods, ${ }^{149} 150$ automation in instrumentation and data analysis (eg, chromatographic peak and quality controls), ${ }^{151152}$ and flat file interface to laboratory information systems to facilitate seamless orderto-result workflows. ${ }^{153}$ Operational factors such as standardised workflow, turnaround time, and comprehensive biocomputational data analysis and storage should also be considered. More details on the current state and further challenges of routinely implementing MSI in the clinical pathology laboratory are highlighted by experts in the field. ${ }^{139} 154$ In summary, future innovations in technology and instrumentation will drive novel clinical applications of MS to the forefront.

\section{Take home messages}

- Mass spectrometry-based clinical applications are expanding into various disciplines of clinical diagnostic laboratories including clinical chemistry, microbiology, and more recently anatomic pathology.

- This review summarises mass spectrometry method principles, instrumentation, and current established clinical applications.

- This review highlights current and emerging mass spectrometry-based applications in anatomic pathology including omics, mass spectrometry imaging, and real-time in vivo techniques.

\section{Handling editor Runjan Chetty.}

Acknowledgements We thank renal pathologist Dr Mei Lin Bissonnette for providing her expertise and input.

Contributors AWSF, VS, AHR and VK contributed to review outline, writing and editing.

Funding The authors have not declared a specific grant for this research from any funding agency in the public, commercial or not-for-profit sectors.

Competing interests None declared.

Patient consent for publication Not required.

Provenance and peer review Commissioned; internally peer reviewed.

ORCID iD

Vathany Kulasingam http://orcid.org/0000-0001-5610-873X

\section{REFERENCES}

1 Jannetto PJ, Fitzgerald RL. Effective use of mass spectrometry in the clinical laboratory. Clin Chem 2016;62:92-8.

2 Strathmann FG, Hoofnagle AN. Current and future applications of mass spectrometry to the clinical laboratory. Am J Clin Pathol 2011;136:609-16.

3 Adaway JE, Keevil BG, Owen LJ. Liquid chromatography tandem mass spectrometry in the clinical laboratory. Ann Clin Biochem 2015;52:18-38.

4 Rolland DCM, Lim MS, Elenitoba-Johnson KSJ. Mass spectrometry and proteomics in hematology. Semin Hematol 2019;56:52-7.

5 Yang Z, Wang S. Recent development in application of high performance liquid chromatography-tandem mass spectrometry in therapeutic drug monitoring of immunosuppressants. J Immunol Methods 2008;336:98-103.

6 Ansermot N, Fathi M, Veuthey J-L, et al. Simultaneous quantification of cyclosporine, tacrolimus, sirolimus and everolimus in whole blood by liquid chromatographyelectrospray mass spectrometry. Clin Biochem 2008;41:728-35.

7 Bardin S, Ottinger JC, Breau AP, et al. Determination of free levels of phenytoin in human plasma by liquid chromatography/tandem mass spectrometry. J Pharm Biomed Anal 2000;23:573-9.

8 Beck 0, Ohman I, Nordgren HK. Determination of lamotrigine and its metabolites in human plasma by liquid chromatography-mass spectrometry. Ther Drug Monit 2006;28:603-7.

9 Blonk MI, van der Nagel BC, Smit LS, et al. Quantification of levetiracetam in plasma of neonates by ultra performance liquid chromatography-tandem mass spectrometry. Journal of Chromatography B 2010;878:675-81.
10 Baietto L, D'Avolio A, Ventimiglia G, et al. Development, validation, and routine application of a high-performance liquid chromatography method coupled with a single mass detector for quantification of itraconazole, voriconazole, and posaconazole in human plasma. Antimicrob Agents Chemother 2010;54:3408-13.

11 Borrey D, Moerman E, Cockx A, et al. Column-switching LC-MS/MS analysis for quantitative determination of testosterone in human serum. Clinica Chimica Acta 2007;382:134-7.

12 Søeborg T, Frederiksen H, Fruekilde P, et al. Serum concentrations of DHEA, DHEAS, $17 \alpha$-hydroxyprogesterone, $\Delta 4$-androstenedione and testosterone in children determined by TurboFlow-LC-MS/MS. Clinica Chimica Acta 2013;419:95-101.

13 Saenger AK, Laha TJ, Bremner DE, et al. Quantification of serum 25-hydroxyvitamin D2 and D3using HPLC-Tandem Mass Spectrometry and examination of reference intervals for diagnosis of vitamin D deficiency. Am J Clin Pathol 2006;125:914-20.

14 Hosogi J, Tanaka H, Fujita K, et al. Lc-Ms/Ms coupled with immunoaffinity extraction for determination of estrone, 17beta-estradiol and estrone 3-sulfate in human plasma. J Chromatogr B Analyt Technol Biomed Life Sci 2010;878:222-7.

15 Kushnir MM, Rockwood AL, Yue B, et al. High sensitivity measurement of estrone and estradiol in serum and plasma using LC-MS/MS. Methods Mol Biol 2010:603:219-28

16 Li W, Li Y-H, Li AC, et al. Simultaneous determination of norethindrone and ethinyl estradiol in human plasma by high performance liquid chromatography with tandem mass spectrometry--experiences on developing a highly selective method using derivatization reagent for enhancing sensitivity. J Chromatogr B Analyt Technol Biomed Life Sci 2005:825:223-32.

17 Etter ML, Eichhorst J, Lehotay DC. Clinical determination of 17-hydroxyprogesterone in serum by LC-MS/MS: comparison to Coat-A-Count RIA method. J Chromatogr B Analyt Technol Biomed Life Sci 2006;840:69-74.

18 Huang W, Kalhorn TF, Baillie M, et al. Determination of free and total cortisol in plasma and urine by liquid chromatography-tandem mass spectrometry. Ther Drug Monit 2007:29:215-24.

19 Van Der Gugten JG, Holmes DT. Quantitation of aldosterone in serum or plasma using liquid chromatography-tandem mass spectrometry (LC-MS/MS). Methods Mol Biol 2016;1378:37-46.

20 de Jong WHA, Graham KS, van der Molen JC, et al. Plasma free metanephrine measurement using automated online solid-phase extraction HPLC tandem mass spectrometry. Clin Chem 2007;53:1684-93.

21 Kushnir MM, Komaromy-Hiller G, Shushan B, et al. Analysis of dicarboxylic acids by tandem mass spectrometry. high-throughput quantitative measurement of methylmalonic acid in serum, plasma, and urine. Clin Chem 2001:47:1993-2002.

22 Lakso H-A, Appelblad P, Schneede J. Quantification of methylmalonic acid in human plasma with hydrophilic interaction liquid chromatography separation and mass spectrometric detection. Clin Chem 2008;54:2028-35.

23 Chace DH, DiPerna JC, Mitchell BL, et al. Electrospray tandem mass spectrometry for analysis of acylcarnitines in dried postmortem blood specimens collected at autopsy from infants with unexplained cause of death. Clin Chem 2001;47:1166-82.

24 Hardy DT, Preece MA, Green A. Determination of plasma free carnitine by electrospray tandem mass spectrometry. Ann Clin Biochem 2001;38:665-70.

25 Anderson NL, Anderson NG, Haines LR, et al. Mass spectrometric quantitation of peptides and proteins using stable isotope standards and capture by anti-peptide antibodies (SISCAPA). J Proteome Res 2004:3:235-44.

26 Hoofnagle AN, Becker JO, Wener MH, et al. Quantification of thyroglobulin, a lowabundance serum protein, by immunoaffinity peptide enrichment and tandem mass spectrometry. Clin Chem 2008;54:1796-804.

27 van der Gugten G, DeMarco ML, Chen LYC, et al. Resolution of spurious immunonephelometric lgG subclass measurement discrepancies by LC-MS/MS. Clin Chem 2018;64:735-42.

28 Mills JR, Barnidge DR, Murray DL. Detecting monoclonal immunoglobulins in human serum using mass spectrometry. Methods 2015;81:56-65.

29 Kohlhagen MC, Barnidge DR, Mills JR, et al. Screening method for M-proteins in serum using nanobody enrichment coupled to MALDI-TOF mass spectrometry. Clin Chem 2016;62:1345-52

30 Wang Z, Dunlop K, Long SR, et al. Mass spectrometric methods for generation of protein mass database used for bacterial identification. Anal Chem 2002:74:3174-82

31 Eigner U, Holfelder M, Oberdorfer K, et al. Performance of a matrix-assisted laser desorption ionization-time-of-flight mass spectrometry system for the identification of bacterial isolates in the clinical routine laboratory. Clin Lab 2009;55:289-96.

32 Gao X, Tan BH, Sugrue RJ, et al. MALDI mass spectrometry for nucleic acid analysis. Top Curr Chem 2013;331:55-77.

33 Vogel N, Schiebel K, Humeny A. Technologies in the whole-genome age: MALDI-TOFbased genotyping. Transfus Med Hemother 2009;36:253-62.

34 Meyer K, Ueland PM. Use of matrix-assisted laser desorption/ionization time-offlight mass spectrometry for multiplex genotyping. Adv Clin Chem 2011;53:1-29.

35 Chace DH, Millington DS, Terada N, et al. Rapid diagnosis of phenylketonuria by quantitative analysis for phenylalanine and tyrosine in neonatal blood spots by tandem mass spectrometry. Clin Chem 1993;39:66-71.

36 Dhillon KS, Bhandal AS, Aznar CP, et al. Improved tandem mass spectrometry (MS MS) derivatized method for the detection of tyrosinemia type I, amino acids and 
acylcarnitine disorders using a single extraction process. Clinica Chimica Acta 2011:412:873-9.

37 Naylor EW, Chace DH. Automated tandem mass spectrometry for mass newborn screening for disorders in fatty acid, organic acid, and amino acid metabolism. J Child Neurol 1999;14 Suppl 1.

38 Jeong J-S, Kim S-K, Park S-R. Amino acid analysis of dried blood spots for diagnosis of phenylketonuria using capillary electrophoresis-mass spectrometry equipped with a sheathless electrospray ionization interface. Anal Bioanal Chem 2013:405:8063-72

39 Seo JY, Park H-D, Kim JW, et al. Steroid profiling for congenital adrenal hyperplasia by tandem mass spectrometry as a second-tier test reduces follow-up burdens in a tertiary care Hospital: a retrospective and prospective evaluation. J Perinat Med 2014:42:121-7.

40 Tellez MR, Mamikunian G, O'Dorisio TM, et al. A single fasting plasma 5-HIAA value correlates with 24-hour urinary 5-HIAA values and other biomarkers in midgut neuroendocrine tumors (nets). Pancreas 2013;42:405-10.

41 Van Der Gugten JG, Wong S, Holmes DT. Quantitation of insulin analogues in serum using immunoaffinity extraction, liquid chromatography, and tandem mass spectrometry. Methods Mol Biol 2016;1378:119-30.

42 Sepiashvili L, Kohlhagen MC, Snyder MR, et al. Direct detection of monoclonal free light chains in serum by use of immunoenrichment-coupled MALDI-TOF mass spectrometry. Clin Chem 2019;65:1015-22.

43 Ketha SS, Singh RJ, Ketha H. Role of mass spectrometry in clinical endocrinology. Endocrinol Metab Clin North Am 2017;46:593-613.

44 Mills JR, Kohlhagen MC, Dasari S, et al. Comprehensive assessment of M-proteins using nanobody enrichment coupled to MALDI-TOF mass spectrometry. Clin Chem 2016;62:1334-44.

45 Hattan SJ, Parker KC, Vestal ML, et al. Analysis and quantitation of glycated hemoglobin by matrix assisted laser desorption/ionization time of flight mass spectrometry. J Am Soc Mass Spectrom 2016;27:532-41.10.1007/s13361-0151316-6

46 Oran PE, Trenchevska O, Nedelkov D, et al. Parallel Workflow for High-Throughput (>1,000 Samples/Day) Quantitative Analysis of Human Insulin-Like Growth Factor 1 Using Mass Spectrometric Immunoassay. PLoS One 2014;9:e92801.

47 Gao J, Meyer K, Borucki K, et al. Multiplex immuno-MALDI-TOF MS for targeted quantification of protein biomarkers and their proteoforms related to inflammation and renal dysfunction. Anal Chem 2018;90:3366-73.

48 Chen B-M, Liang Y-Z, Chen X, et al. Quantitative determination of azithromycin in human plasma by liquid chromatography-mass spectrometry and its application in a bioequivalence study. J Pharm Biomed Anal 2006;42:480-7.

49 Gu Y, Wang G, Sun J. Simultaneous determination of erythromycin ethylsuccinate and its metabolite erythromycin in human plasma using liquid chromatographyelectrospray ionization mass spectrometry for clinical study. J Pharm Biomed Anal 2006;40:737-43.

50 Bonaparte E, Pesenti C, Fontana L, et al. Molecular profiling of lung cancer specimens and liquid biopsies using MALDI-TOF mass spectrometry. Diagn Pathol 2018; $13: 7$

51 DeMarco ML, Ford BA. Beyond identification: emerging and future uses for MALDI-TOF mass spectrometry in the clinical microbiology laboratory. Clin Lab Med 2013:33:611-28.

52 Duncan MW, Nedelkov D, Walsh R, et al. Applications of MALDI mass spectrometry in clinical chemistry. Clin Chem 2016;62:134-43.

53 Duncan M, DeMarco ML. Maldi-Ms: emerging roles in pathology and laboratory medicine. Clin Mass Spectrom 2019;13:1-4.

54 Jain D, Green JA, Bastacky S, et al. Membranoproliferative glomerulonephritis: the role for laser microdissection and mass spectrometry. Am J Kidney Dis 2014;63:324-8.10.1053/j.ajkd.2013.09.007

55 Sethi S, Vrana JA, Theis JD, et al. Mass spectrometry based proteomics in the diagnosis of kidney disease. Curr Opin Nephrol Hypertens 2013;22:273-80.

56 Herrera GA, Turbat-Herrera EA. Ultrastructural immunolabeling in the diagnosis of monoclonal light-and heavy-chain-related renal diseases. Ultrastruct Pathol 2010;34:161-73

57 Sethi S, Theis JD, Vrana JA, et al. Laser microdissection and proteomic analysis of amyloidosis, cryoglobulinemic GN, fibrillary GN, and immunotactoid glomerulopathy. CJASN 2013;8:915-21.

58 Sethi S, Fervenza FC, Rajkumar SV. Spectrum of manifestations of monoclonal gammopathy-associated renal lesions. Curr Opin Nephrol Hypertens 2016:25:127-37

59 Sethi S, Theis JD, Leung N, et al. Mass Spectrometry-Based proteomic diagnosis of renal immunoglobulin heavy chain amyloidosis. CJASN 2010:5:2180-7.

60 Theis JD, Dasari S, Vrana JA, et al. Shotgun-proteomics-based clinical testing for diagnosis and classification of amyloidosis. J. Mass Spectrom. 2013:48:1067-77.

61 Sipe JD, Benson MD, Buxbaum JN, et al. Amyloid fibril protein nomenclature: 2012 recommendations from the nomenclature Committee of the International Society of amyloidosis. Amyloid 2012;19:167-70.

62 Sipe JD, Benson MD, Buxbaum JN, et al. Amyloid fibril proteins and amyloidosis: chemical identification and clinical classification International Society of amyloidosis 2016 nomenclature guidelines. Amyloid 2016;23:209-13.
63 Bridoux F, Leung N, Hutchison CA, et al. Diagnosis of monoclonal gammopathy of renal significance. Kidney Int 2015;87:698-711.

64 Vrana JA, Gamez JD, Madden BJ, et al. Classification of amyloidosis by laser microdissection and mass spectrometry-based proteomic analysis in clinical biopsy specimens. Blood 2009;114:4957-9

65 Saha A, Theis JD, Vrana JA, et al. AA amyloidosis associated with hepatitis B. Nephrol Dial Transplant 2011;26:2407-12.

66 Sethi S, Theis JD, Shiller SM, et al. Medullary amyloidosis associated with apolipoprotein A-IV deposition. Kidney Int 2012;81:201-6.

67 Nasr SH, Said SM, Valeri AM, et al. The diagnosis and characteristics of renal heavychain and heavy/light-chain amyloidosis and their comparison with renal light-chain amyloidosis. Kidney Int 2013;83:463-70.

68 Mollee P, Boros S, Loo D, et al. Implementation and evaluation of amyloidosis subtyping by laser-capture microdissection and tandem mass spectrometry. Clin Proteomics 2016:13:30.

69 Park J, Lee GY, Choi J-0, et al. Development and validation of mass spectrometry-based targeted analysis for amyloid proteins. Proteomics Clin Appl 2018;12:e1700106.

70 Leung N, Bridoux F, Batuman V, et al. The evaluation of monoclonal gammopathy of renal significance: a consensus report of the International kidney and monoclonal gammopathy Research Group. Nat Rev Nephrol 2019;15:45-59.

71 Sethi S, Vrana JA, Theis JD, et al. Laser microdissection and mass spectrometrybased proteomics AIDS the diagnosis and typing of renal amyloidosis. Kidney Int 2012;82:226-34.

72 Nasr SH, Fogo AB. New developments in the diagnosis of fibrillary glomerulonephritis. Kidney Int 2019.

73 Nasr SH, Valeri AM, Cornell LD, et al. Fibrillary glomerulonephritis: a report of 66 cases from a single institution. CJASN 2011;6:775-84.

74 Alexander MP, Dasari S, Vrana JA, et al. Congophilic fibrillary glomerulonephritis: a case series. Am J Kidney Dis 2018;72:325-36.

75 Dasari S, Alexander MP, Vrana JA, et al. Dnaj heat shock protein family B member 9 is a novel biomarker for fibrillary GN. J Am Soc Nephrol 2018;29:51-6.

76 Nasr SH, Vrana JA, Dasari S, et al. DNAJB9 is a specific immunohistochemical marker for fibrillary glomerulonephritis. Kidney Int Rep 2018:3:56-64

77 Andeen NK, Yang H-Y, Dai D-F, et al. Dnaj homolog subfamily B member 9 is a putative autoantigen in fibrillary GN. J Am Soc Nephrol 2018;29:231-9.

78 Nasr SH, Sirac C, Bridoux F, et al. Heavy chain fibrillary glomerulonephritis: a case report. Am J Kidney Dis 2019;74:276-80.

79 Nasr SH, Dasari S, Lieske JC, et al. Serum levels of DNAJB9 are elevated in fibrillary glomerulonephritis patients. Kidney Int 2019;95:1269-72.

80 Nasr SH, Fidler ME, Cornell LD, et al. Immunotactoid glomerulopathy: clinicopathologic and proteomic study. Nephrol Dial Transplant 2012;27:4137-46.

81 Leung N, Drosou ME, Nasr SH. Dysproteinemias and glomerular disease. CJASN 2018;13:128-39.

82 Bridoux F, Hugue V, Coldefy 0 , et al. Fibrillary glomerulonephritis and immunotactoid (microtubular) glomerulopathy are associated with distinct immunologic features. Kidney Int 2002:62:1764-75.

83 Yahata M, Nakaya I, Takahashi S, et al. Proliferative glomerulonephritis with monoclonal IgM deposits without Waldenström's macroglobulinemia: case report and review of the literature. Clin Nephrol 2012;77:254-60.

84 Soares SM, Lager DJ, Leung $\mathrm{N}$, et al. A proliferative glomerulonephritis secondary to a monoclonal IgA. Am J Kidney Dis 2006;47:342-9.

85 Alpers CE, Tu W-H, Hopper J, et al. Single light chain subclass (kappa chain) immunoglobulin deposition in glomerulonephritis. Hum Pathol 1985;16:294-304.

86 Nasr SH, Satoskar A, Markowitz GS, et al. Proliferative glomerulonephritis with monoclonal IgG deposits. J Am Soc Nephrol 2009;20:2055-64.

87 Royal V, Quint P, Leblanc M, et al. Igd heavy-chain deposition disease: detection by laser microdissection and mass spectrometry. J Am Soc Nephrol 2015;26:784-90.

88 Larsen CP, Messias NC, Walker PD, et al. Membranoproliferative glomerulonephritis with masked monotypic immunoglobulin deposits. Kidney Int 2015;88:867-73.

89 Lloyd IE, Gallan A, Huston HK, et al. C3 glomerulopathy in adults: a distinct patient subset showing frequent association with monoclonal gammopathy and poor renal outcome. Clin Kidney J 2016:9:794-9.

90 Kasagi T, Nobata H, Suzuki K, et al. Light chain deposition disease diagnosed with laser micro-dissection, liquid chromatography, and tandem mass spectrometry of nodular glomerular lesions. Intern. Med. 2017;56:61-6.

91 Sethi S, Zand L, Leung N, et al. Membranoproliferative glomerulonephritis secondary to monoclonal gammopathy. Clin J Am Soc Nephrol 2010;5:770-82.

92 Smith RJH, Appel GB, Blom AM, et al. C3 glomerulopathy — understanding a rare complement-driven renal disease. Nat Rev Nephrol 2019;15:129-43.

93 Sethi S, Gamez JD, Vrana JA, et al. Glomeruli of dense deposit disease contain components of the alternative and terminal complement pathway. Kidney Int 2009:75:952-60.

94 Sethi S, Vrana JA, Fervenza FC, et al. Characterization of C3 in C3 glomerulopathy. Nephrol Dial Transplant 2017;32:459-65.

95 Sethi S, Sukov WR, Zhang Y, et al. Dense deposit disease associated with monoclonal gammopathy of undetermined significance. Am J Kidney Dis 2010;56:977-82. 
96 Zand L, Kattah A, Fervenza FC, et al. C3 glomerulonephritis associated with monoclonal gammopathy: a case series. Am J Kidney Dis 2013;62:506-14.

97 Meri S, Koistinen V, Miettinen A, et al. Activation of the alternative pathway of complement by monoclonal lambda light chains in membranoproliferative glomerulonephritis. J Exp Med 1992;175:939-50.

98 Jokiranta TS, Solomon A, Pangburn MK, et al. Nephritogenic lambda light chain dimer: a unique human miniautoantibody against complement factor $\mathrm{H}$. J Immunol 1999:163:4590-6.

99 Sethi S, Rajkumar SV. Monoclonal gammopathy-associated proliferative glomerulonephritis. Mayo Clin Proc 2013;88:1284-93.

100 Blanc C, Togarsimalemath SK, Chauvet S, et al. Anti-Factor H autoantibodies in C3 glomerulopathies and in atypical hemolytic uremic syndrome: one target, two diseases. J Immunol 2015;194:5129-38.

101 Kriegsmann J, Kriegsmann M, Casadonte R. Maldi TOF imaging mass spectrometry in clinical pathology: a valuable tool for cancer diagnostics (review). Int J Oncol 2015;46:893-906.

102 Ifa DR, Eberlin LS. Ambient ionization mass spectrometry for cancer diagnosis and surgical margin evaluation. Clin Chem 2016;62:111-23.

103 Prideaux B, Stoeckli M. Mass spectrometry imaging for drug distribution studies. J Proteomics 2012;75:4999-5013.

104 Sun N, Walch A. Qualitative and quantitative mass spectrometry imaging of drugs and metabolites in tissue at therapeutic levels. Histochem Cell Biol 2013;140:93-104.

105 Cole LM, Clench MR. Mass spectrometry imaging tools in oncology. Biomark Med 2015;9:863-8

106 Caprioli RM, Farmer TB, Gile J. Molecular imaging of biological samples: localization of peptides and proteins using MALDI-TOF MS. Anal Chem 1997;69:4751-60.

107 van Remoortere A, van Zeijl RJ, van den Oever N, et al. Maldi imaging and profiling MS of higher mass proteins from tissue. J Am Soc Mass Spectrom 2010;21:1922-9.

108 Longuespée R, Boyon C, Castellier C, et al. The C-terminal fragment of the immunoproteasome PA28S (reg alpha) as an early diagnosis and tumor-relapse biomarker: evidence from mass spectrometry profiling. Histochem Cell Biol 2012;138:141-54.

109 Yang J, Caprioli RM. Matrix sublimation/recrystallization for imaging proteins by mass spectrometry at high spatial resolution. Anal Chem 2011;83:5728-34.

110 Gustafsson OJR, Eddes JS, Meding S, et al. Matrix-assisted laser desorption/ ionization imaging protocol for in situ characterization of tryptic peptide identity and distribution in formalin-fixed tissue. Rapid Commun. Mass Spectrom. 2013:27:655-70.

111 Gustafsson JOR, Oehler MK, McColl SR, et al. Citric acid antigen retrieval (CaAR) for tryptic peptide imaging directly on archived formalin-fixed paraffin-embedded tissue. J Proteome Res 2010;9:4315-28.

112 Bonnel D, Longuespee R, Franck J, et al. Multivariate analyses for biomarkers hunting and validation through on-tissue bottom-up or in-source decay in MALDI-MSI: application to prostate cancer. Anal Bioanal Chem 2011;401:149-65.

113 Longuespee R, Gagnon H, Boyon C, et al. Proteomic analyses of serous and endometrioid epithelial ovarian cancers - Cases studies - Molecular insights of a possible histological etiology of serous ovarian cancer. Proteomics Clin Appl 2013;7:337-54.

114 Gode D, Volmer DA. Lipid imaging by mass spectrometry - a review. Analyst 2013;138:1289-315

115 Gorzolka K, Walch A. Maldi mass spectrometry imaging of formalin-fixed paraffinembedded tissues in clinical research. Histol Histopathol 2014;29:1365-76.

116 Longuespée R, Casadonte R, Kriegsmann M, et al. Maldi mass spectrometry imaging: a cutting-edge tool for fundamental and clinical histopathology. Proteomics Clin App/ 2016;10:701-19.

117 Baker TC, Han J, Borchers CH. Recent advancements in matrix-assisted laser desorption/ionization mass spectrometry imaging. Curr Opin Biotechnol 2017:43:62-9.

118 Shimma S, Kumada H-O, Taniguchi H, et al. Microscopic visualization of testosterone in mouse testis by use of imaging mass spectrometry. Anal Bioanal Chem 2016;408:7607-15

119 Wu Q, Comi TJ, Li B, et al. On-Tissue derivatization via electrospray deposition for matrix-assisted laser desorption/ionization mass spectrometry imaging of endogenous fatty acids in rat brain tissues. Anal Chem 2016;88:5988-95.

120 Shariatgorji M, Nilsson A, Goodwin RJA, et al. Direct targeted quantitative molecular imaging of neurotransmitters in brain tissue sections. Neuron 2014;84:697-707.

121 Holst S, Heijs B, de Haan N, et al. Linkage-Specific in Situ Sialic Acid Derivatization for N-Glycan Mass Spectrometry Imaging of Formalin-Fixed Paraffin-Embedded Tissues. Anal Chem 2016:88:5904-13.

122 Vens-Cappell S, Kouzel IU, Kettling H, et al. On-Tissue phospholipase C digestion for enhanced MALDI-MS imaging of neutral glycosphingolipids. Anal Chem 2016;88:5595-9.

123 Dreisewerd K. Recent methodological advances in MALDI mass spectrometry. Anal Bioanal Chem 2014:406:2261-78.

124 Stübiger G, Belgacem 0. Analysis of lipids using 2,4,6-trihydroxyacetophenone as a matrix for MALDI mass spectrometry. Anal Chem 2007:79:3206-13.
125 Woods AS, Huestis MA. A study of peptide-peptide interaction by matrix-assisted laser desorption/ionization. J Am Soc Mass Spectrom 2001;12:88-96.

126 Zehl M, Allmaier G, Allmaier G. Investigation of sample preparation and instrumenta parameters in the matrix-assisted laser desorption/ionization time-of-flight mass spectrometry of noncovalent peptide/peptide complexes. Rapid Commun Mass Spectrom 2003; 17:1931-40.

127 Armstrong DW, Zhang L-K, He L, et al. Ionic liquids as matrixes for matrix-assisted laser desorption/ionization mass spectrometry. Anal Chem 2001;73:3679-86.

128 Bouschen W, Schulz 0, Eikel D, et al. Matrix vapor deposition/recrystallization and dedicated spray preparation for high-resolution scanning microprobe matrix-assisted laser desorption/ionization imaging mass spectrometry (SMALDI-MS) of tissue and single cells. Rapid Commun. Mass Spectrom. 2010;24:355-64.

129 Wang X, Han J, Yang J, et al. Matrix coating assisted by an electric field (MCAEF) for enhanced tissue imaging by MALDI-MS. Chem. Sci. 2015;6:729-38.

130 Wang $\mathrm{X}$, Han J, Hardie DB, et al. The use of matrix coating assisted by an electric field (MCAEF) to enhance mass spectrometric imaging of human prostate cance biomarkers. J Mass Spectrom 2016:51:86-95.

131 Gemperline E, Rawson S, Li L. Optimization and comparison of multiple MALDI matrix application methods for small molecule mass spectrometric imaging. Anal Chem 2014;86:10030-5.

132 Hankin JA, Barkley RM, Murphy RC. Sublimation as a method of matrix application for mass spectrometric imaging. J Am Soc Mass Spectrom 2007;18:1646-52.

133 Grove KJ, Frappier SL, Caprioli RM. Matrix pre-coated MALDI MS targets for small molecule imaging in tissues. J Am Soc Mass Spectrom 2011:22:192-5.

134 Yang J, Caprioli RM. Matrix precoated targets for direct lipid analysis and imaging of tissue. Anal Chem 2013:85:2907-12.

135 Lanni EJ, Dunham SJB, Nemes P, et al. Biomolecular imaging with a C60SIMS/MALDI dual ion source hybrid mass spectrometer: instrumentation, matrix enhancement, and single cell analysis. J Am Soc Mass Spectrom 2014;25:1897-907.

136 Lockyer NP. Secondary ion mass spectrometry imaging of biological cells and tissues. Methods Mol Biol 2014;1117:707-32.

137 Addie RD, Balluff B, Bovée JVMG, et al. Current state and future challenges of mass spectrometry imaging for clinical research. Anal Chem 2015;87:6426-33.

138 Perez CJ, Bagga AK, Prova SS, et al. Review and perspectives on the applications of mass spectrometry imaging under ambient conditions. Rapid Commun Mass Spectrom 2018.

139 Kriegsmann J, Casadonte R, Kriegsmann K, et al. Mass spectrometry in pathology vision for a future workflow. Pathol Res Pract 2018:214:1057-63.

140 Schäfer K-C, Dénes J, Albrecht K, et al. In vivo, in situ tissue analysis using rapid evaporative ionization mass spectrometry. Angewandte Chemie International Edition 2009:48:8240-2.

141 Golf O, Strittmatter N, Karancsi T, et al. Rapid evaporative ionization mass spectrometry imaging platform for direct mapping from bulk tissue and bacteria growth media. Anal Chem 2015;87:2527-34

142 Balog J, Sasi-Szabó L, Kinross J, et al. Intraoperative tissue identification using rapid evaporative ionization mass spectrometry. Sci Trans/ Med 2013;5:194ra93.

143 Balog J, Szaniszlo T, Schaefer K-C, et al. Identification of biological tissues by rapid evaporative ionization mass spectrometry. Anal Chem 2010;82:7343-50.

144 Balog J, Sasi-Szabo L, Kinross J, et al. Intraoperative tissue identification using rapid evaporative ionization mass spectrometry. Sci Transl Med 2013;5:194ra93.

145 Alexander J, Gildea L, Balog J, et al. A novel methodology for in vivo endoscopic phenotyping of colorectal cancer based on real-time analysis of the mucosal lipidome: a prospective observational study of the iKnife. Surg Endosc 2017:31:1361-70.

146 Zhang J, Rector J, Lin JQ, et al. Nondestructive tissue analysis for ex vivo and in vivo cancer diagnosis using a handheld mass spectrometry system. Sci Trans/ Med 2017;9:eaan3968

147 Lynch KL. Clsi C62-A: a new standard for clinical mass spectrometry. Clin Chem 2016:62:24-9.

148 Lynch KL. Accreditation and quality assurance for clinical liquid ChromatographyMass spectrometry laboratories. Clin Lab Med 2018;38:515-26.

149 Vesper HW, Botelho JC. Standardization of testosterone measurements in humans. J Steroid Biochem Mol Biol 2010;121:513-9.

150 Wise SA, Phinney KW, Tai SS-C, et al. Baseline assessment of 25-hydroxyvitamin $D$ assay performance: a vitamin D standardization program (VDSP) interlaboratory comparison study. J AOAC Int 2017;100:1244-52

151 Dickerson JA, Schmeling M, Hoofnagle AN, et al. Design and implementation of software for automated quality control and data analysis for a complex LC/MS/MS assay for urine opiates and metabolites. Clinica Chimica Acta 2013:415:290-4.

152 Vicente FB, Lin DC, Haymond S. Automation of chromatographic peak review and order to result data transfer in a clinical mass spectrometry laboratory. Clinica Chimica Acta 2019:498:84-9.

153 Holmes DT. Flat file interface your mass spectrometer to the laboratory information system with R 2016.

154 Leung F, Eberlin LS, Schwamborn K, et al. Mass spectrometry-based tissue imaging: the next frontier in clinical diagnostics? Clin Chem 2019:65:510-3. 